\title{
Match injury incidence during the Super Rugby tournament is high: a prospective cohort study over five seasons involving 93641 player- hours
}

Martin P Schwellnus, Esme Jordaan, Charl Janse van Rensburg, Helen Bayne, Wayne Derman, Clint Readhead, Rob Collins, Alan Kourie, Jason Suter and Org Strauss

\begin{abstract}
:
Objectives To determine the incidence and nature of injuries in the Super Rugby tournament over a 5year period.

Methods 482 male professional rugby union players from six South African teams participating in the Super Rugby tournament were studied (1020 player-seasons). Medical staff of participating teams (2012-2016 tournaments) recorded all time loss injuries (total injuries and match injuries) and exposure hours (93 641 total playing hours; 8032 match hours). Injury incidence, injured player proportion, severity (time lost), anatomical location, tissue type and activity/phase during which injury occurred are reported.

Results The overall incidence of match injuries (per 1000 player-hours; 95\% CI) for each year was as follows: 2012 (83.3; 69.4-99.2); 2013 (115.1; 98.7-133.5); 2014 (95.9; 80.8-113.1), 2015 (112.3; 96.6-129.9) and 2016 (93.2; 79.9-107.9). The injured player proportion for each year was as follows: 2012 (54.6\%); 2013 (49.4\%); 2014 (52.0\%); 2015 (50.0\%); and 2016 (39.8\%). The thigh, knee, head/face and shoulder/ clavicle are the most frequently injured locations, and muscle/tendon and joint/ligament injuries account for the majority of injuries. Most injuries (79\%) occur in contact situations, in particular during a tackle (54\%).

Conclusion The incidence of match injuries and the injured player proportion in South African teams competing in the Super Rugby tournament is high. Match injury incidence is consistently higher than previously reported for senior male rugby players at elite/ professional level. Targeted risk management strategies are therefore needed in the Super Rugby tournament to manage risk of injury.
\end{abstract}

\section{Introduction}

Rugby union is a contact sport involving frequent high-impact collisions. As a result, it has one of the highest rates of injury incidence among team sports. ${ }^{1}$ Injuries to rugby players have a negative effect on player welfare and team success. ${ }^{2}$

The first step towards instituting an effective injury prevention programme is establishing a comprehensive understanding of the magnitude and nature of the injury problem.3 A metaanalysis published in 2013 reported a match and training injury incidence of 81 and 3 per 1000 
player-hours, respectively, by combining data from 10 epidemiological studies in senior men's professional rugby union. ${ }^{1}$ However, the analysis was weighted towards the largest and longest running study (the England Rugby Premiership Injury Surveillance Project), 4 which may differ from rugby played in other regions or competitions.

Two subsequent studies, each conducted over a single season, have investigated injury incidence during the Super Rugby tournament where matches are played in Australia, New Zealand and South Africa. In five South African teams during the 2012 season, the match injury incidence was similar to the meta-analysis (83 per 1000 player-hours), but presented a lower overall severity profile. 5 In five Australian teams during the 2014 season, the match injury incidence was lower (66 per 1000 player-hours), but the number of severe injuries (resulting in more than 28 days of time loss) was higher. 6 These two studies are however limited because they documented injuries in only a single season.

World Rugby, the international governing body for rugby union, periodically introduces changes to the laws of the game to enhance the playing and spectating experience and improve player welfare. Such law changes likely alter team strategy and player demands and may affect injury patterns over time.7 There is therefore a need to conduct longitudinal injury epidemiology studies over a number of seasons in order to analyse changes in injury profiles. Currently, such data are only available for one competition-the England Rugby Premiership. 8 Therefore, the aim of this research study was to describe the incidence and nature of injuries in the South African teams competing in the Super Rugby tournament over five seasons.

\section{Methods}

This prospective cohort study involved 482 players from six South African teams in the Super Rugby tournament over 5 years (2012-2016). At least five teams participated in the study in each year. Each of the team physicians accompanying the teams was provided with detailed information about the study. The team physicians then briefed all of the players on the study details. Informed consent was obtained from the players through the team physicians. Players were not involved in the planning of the study.

Team physicians of the participating teams collected dataduring the tournament eachyear using an electronic system that had been custom designed for the study. 


\begin{tabular}{|c|c|c|c|}
\hline & All players & Forwards & Backs \\
\hline \multicolumn{4}{|l|}{2012 season } \\
\hline Number of plyyers & 152 & 85 & 67 \\
\hline Total player-hours & 17340 & 9248 & 8092 \\
\hline Tralning hours & 15828 & 8442 & 7386 \\
\hline Match hours & 1512 & 806 & 706 \\
\hline \multicolumn{4}{|l|}{2013 season } \\
\hline Number of plzyers & 180 & 100 & 80 \\
\hline Total player-hours & 17512 & 9951 & 7560 \\
\hline Tralning hours & 15992 & 9141 & 6851 \\
\hline Match hours & 1520 & 811 & 709 \\
\hline \multicolumn{4}{|l|}{2014 season } \\
\hline Number of players & 196 & 110 & 85 \\
\hline Total player-hours & 15517 & 8383 & 7134 \\
\hline Tralning hours & 14037 & 7594 & 6443 \\
\hline Match hours & 1480 & 789 & 691 \\
\hline \multicolumn{4}{|l|}{2015 season } \\
\hline Number of plzyers & 218 & 120 & 98 \\
\hline Total player-hours & 18924 & 10358 & 8566 \\
\hline Tralning hours & 17304 & 9494 & 7810 \\
\hline Match hours & 1620 & 864 & 756 \\
\hline \multicolumn{4}{|l|}{2016 season } \\
\hline Number of players & 274 & 158 & 116 \\
\hline Total player-hours & 24348 & 14158 & 10191 \\
\hline Training hours & 22448 & 13144 & 9304 \\
\hline Match hours & 1900 & 1013 & 887 \\
\hline \multicolumn{4}{|l|}{ Total (all five seasons) } \\
\hline Number of plzyers & 1020 & 573 & 447 \\
\hline Total player-hours & 93641 & 52098 & 41542 \\
\hline Tralning hours & 85609 & 47815 & 37793 \\
\hline Match hours & 8032 & 4283 & 3749 \\
\hline
\end{tabular}




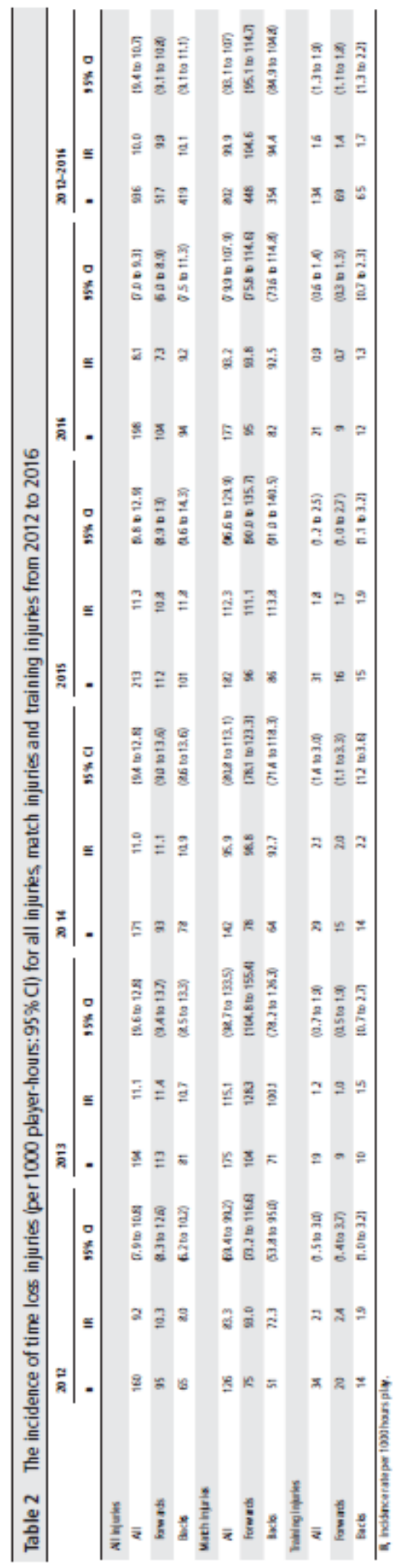

On a daily basis, the team physician could record the number of players in the squad, type of day (rest day, training day or match day) and team training hours. 
Injuries were defined according to the 'Consensus Statement on Injury Definitions and Data Collection Procedures for Studies of Injuries in Rugby Union'. 9 The team physician recorded both medical attention injuries (requiring medical intervention but not resulting in loss of training or match play $>1$ day) and time loss injuries (preventing playing in matches or training for $>1$ day). The time (days) lost for each injury was estimated by the team physician at the time of entry of the injury data, based on their substantive clinical experience. In 2012, where some data on anticipated days lost were missing, the principal investigator (MPS) estimated the total days lost from the diagnosis made by the team physician. We did not obtain the actual days lost for each injury in this study. For the purposes of comparison with other studies, only time loss injuries will be reported in this manuscript.

The following additional injury details were recorded: main player position (forwards, backs), training or match injury, the mechanism of injury, the anatomical location of the injury, the type of injury (muscle/tendon, joint/ligament, skin, bone, brain/ central nervous system (CNS)) and the severity of the injury (days lost from training or matches, estimated at the time of diagnosis). The severity of time loss injuries was subdivided into

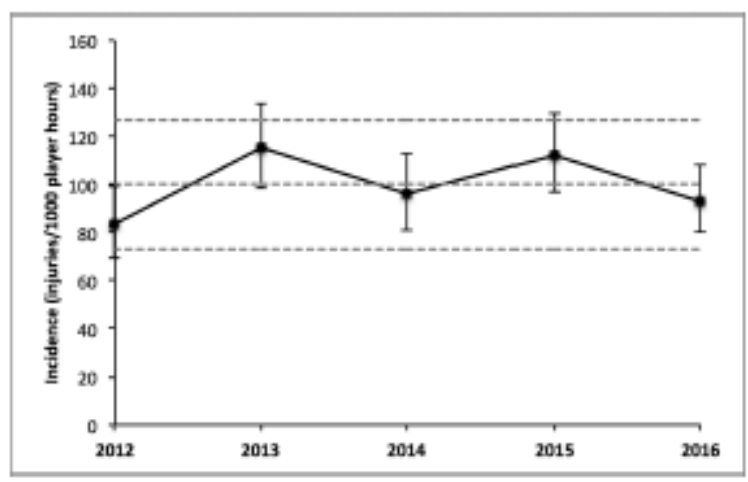

Flgure 1 The Incldence (per 1000 match player-hours) of time loss match injurles from 2012 to 2016 (dashed lines: mean \pm 2 SD).

the following categories, according to the number of estimated days that players were unable to train or play matches: minimal ( $2-3$ days), mild (4-7 days), moderate ( $8-28$ days) and severe (>28 days). 9 More than one injury could be recorded and two injuries in the same player were recorded as separate injuries. The electronic data collection system allowed the research team to monitor data input (logging in and entering data) on a daily basis. This allowed the research team to contact team physicians if data entry was not done regularly.

\section{Statistical Analysis}

Team-based match and training hours were calculated from the data recorded by the team physician. Daily training player-hours for each team were calculated as the number of team training hours $x$ the number of players in the team on that day. It was assumed that all players in the team participated in the entire training session. The total training player-hours were calculated as the sum ofall thedailytraining player-hours for each team in each year. 
A total of 422 Super Rugby matches were played by the participating teams during the surveillance period. Data from 14 matches (6 in 2013 and 8 in 2014) were not available and therefore injury and exposure data from missing matches (3.3\%) were not included in this analysis. The usual total duration of a rugby union match is $80 \mathrm{~min}$ (1.33 hours of play). Match player-hours for one team playing a match were calculated as $1.33 \times 15$ players ( 20 match player-hours). The total match player-hours for a team were calculated as $20 \times$ the number of match days for a team in each year. An adjustment to this calculation was made for one team in 2012, where 18/34 players consented to participate in the study. 5 The total number of players per season, player-hours (all, training, match) for all players, as well as main player positions (forwards and backs) is shown in table 1.

Data, recorded per team, included the total number of injuries sustained, the total number of players injured, the number of match and training injuries, the number of injuries for forwards and backs, number of injuries per severity category, main and specific anatomical location, tissue type and mechanism. The incidence of injury was calculated as injuries per 1000 player-hours of training (training injuries) and match play (match injuries). This enables comparison to the incidence of injuries during matches (match player-hours) to training (training play- er-hours) reported in other studies and followed the international guidelines for injury reporting in rugby union. 9 The proportion of injured players (injured player proportion, IPP) (also known as a period prevalence) was calculated as the per cent of players injured during the tournament. In addition, the IPP for different grades of injury severity was also reported. 5 The burden of match injuries in the 5-year period was calculated as days lost per 1000 match hours.

Standard descriptive statistical analyses were conducted. For injuries, these include numbers, proportions/percentages and incidences (including exact 95\% CIs) of injuries in the total sample as well as subgroups according to matches versus training, main player position, main and specific anatomical location of injury, injury severity categories and activity/phase of play.

\section{Results}

\section{Incidence of all time loss injuries (all, match and training injuries)}

The number of players who participated in the study was 482, and the players in each season was 152 (2012), 180 (2013), 196 (2014), 218 (2105) and 274 (2016), for a combined total of 1020 player-seasons (players participated in multiple years). A total of 93641 player-hours (85 609 training player-hours and 8032 match player-hours) were monitored over the five seasons with match player-hours comprising less than $10 \%$ of the total player-hours (varying from $7.8 \%$ to $9.5 \%$ ). In total 936 time loss injuries were recorded, of which 802 (85.7\%) occurred during matches and 134 (14.3\%) occurred during training. The overall incidence of time loss injuries was 10.0 (95\% CI 9.4 to 10.7) per 1000 player-hours. The incidence of match injuries was significantly greater (99.9; $95 \%$ CI 93.1 to 107.0 ) than training injuries (1.6; $95 \% \mathrm{CI} 1.3$ to 1.9). There were no significant differences in injury incidence between forwards and backs (table 2). Throughout the surveillance period, the incidence of match injuries has remained within two SDs of the overall mean (99.9 per 1000 player-hours, SD 13.4, upper limit 126.9, lower limit 73.1) (figure1). 


\section{Injured player proportion}

The annual IPP ranged from 40\% in 2016 to 55\% in 2012, with an overall average proportion of $48 \%$ (table 3 ). Over the 5 years, $8 \%$ of players suffered a minimal injury, $12 \%$ suffered

\begin{tabular}{|c|c|c|c|c|c|c|c|c|c|c|c|c|}
\hline & \multicolumn{2}{|c|}{2012} & \multicolumn{2}{|c|}{2013} & \multicolumn{2}{|c|}{2014} & \multicolumn{2}{|c|}{2015} & \multicolumn{2}{|c|}{2016} & \multicolumn{2}{|c|}{ 2012-2016 } \\
\hline & n & $\%$ & $\mathbf{n}$ & $\%$ & $\mathrm{n}$ & $\%$ & $\mathrm{n}$ & $\%$ & $\mathbf{n}$ & $\%$ & $\mathbf{n}$ & $\%$ \\
\hline Any Injury & 83 & 54.6 & 89 & 49.4 & 102 & 52.0 & 109 & 50.0 & 109 & 39.8 & 492 & 487 \\
\hline Mirilmal (2-3 days) & 13 & 8.6 & 12 & 6.7 & 16 & 8.2 & 15 & 6.9 & 23 & 8.4 & 79 & 7.7 \\
\hline MIld (4-7 days) & 17 & 11.2 & 23 & 128 & 31 & 15.8 & 36 & 16.5 & 15 & 5.5 & 122 & 12.0 \\
\hline Moderate (8-28 day5) & 31 & 20.4 & 32 & 17.8 & 32 & 16.3 & 37 & 17.0 & 39 & 14.2 & 171 & 16.8 \\
\hline Severe (>28days) & 22 & 14.5 & $n$ & 122 & 23 & 11.7 & 21 & 9.6 & 32 & 11.7 & 120 & 11.8 \\
\hline
\end{tabular}

a mild injury, $17 \%$ suffered a moderate injury and $12 \%$ (one in eight players starting the tournament) suffered a severe injury resulting in at least 28 days of time loss.

\section{Injury severity and burden}

Injury severity was reported for 925 (798 match and 127 training) injuries (table 4). Over the five seasons, the largest proportion of all injuries was minimal (33\%), followed by mild (28\%), moderate (26\%) and severe (14\%). The same pattern was observed for match injuries (minimal 33\%, mild 29\%, moderate $25 \%$ and severe $13 \%$ ) and training injuries (minimal $31 \%$, mild $27 \%$, moderate $28 \%$ and severe $15 \%$ ). Match injury incidence of minimal injury was 40.0 (95\% CI 35.3 to 45.1), mild injury was 34.8 (95\% CI 30.5 to 39.3), moderate injury was 30.9 (95\% CI 26.8 to 35.4 ) and severe injury was 16.2 (95\% CI 13.1 to 19.5). The match injury burden (IB: days lost per 1000 match hours) over the 5-year period was 1796 (95\% CI 1767 to 1826) with a tendency for the match IB to increase over the 5 -year period(figure 2 ).

Main anatomical location (all players, forwards and backs) The majority of the match injuries in all players occurred in the lower limb (50\%), followed by the upper limb (24\%). Less commonly the head/neck region (16\%) and the trunk (11\%) were affected. The same pattern was observed for match injuries in forwards (lower limb $45 \%$, upper limb 25\%, head/neck region $17 \%$ and trunk 13\%) and backs (lower limb 56\%, upper limb 23\%, head/neck region $14 \%$ and trunk $8 \%$ ) (table 5 ).

\section{Specific anatomical structure}

The injuries with the highest incidence over the 5 -year period were to the thigh $(13.1 ; 95 \% \mathrm{CI}$ 10.7 to 15.8 ), knee (13.1; 95\% CI 10.7 to 15.8 ), shoulder/clavicle (12.9; $95 \%$ CI 10.6 to 15.7 ) and head/face (11.7; 95\% CI 9.5 to 14.3 ) (table 6). The incidence of head/face injuries increased from 2013 (5.3; 95\% CI 2.3 to 10.4 ) to 2014 (13.5; 95\% CI 8.3 to 20.9 ) and was the most common injury in 2014 and 2016. 


\section{Type of injuries}

Muscle and tendon injuries consistently accounted for the largest proportion of matchinjuries in the 5 -year period (47\%), followed by joint/ligament injuries (32\%). These types of injuries also occur at a substantially higher incidence rate (muscle/tendon: $46.6,95 \%$ CI 42.0 to 51.5; joint/ligament: 31.9 ; $95 \%$ CI 28.1 to 36.0) than those affecting skin, bone or brain/CNS (table 7). The incidence of injury to the brain/CNS increased from $2013(2.6 ; 95 \% \mathrm{CI} 0.7$ to 6.7$)$ to 2014 (10.8; 95\% CI 6.2 to 17.6) with no significant change in 2015 and 2016. Of the 66 injuries classified as brain/CNS, 60 were concussion and the incidence of concussion showed a similar increase from 2013 to 2014 with no significant change in 2015 and 2016 (table 7).

\section{Mechanism of injuries}

Over the five seasons, $79 \%$ (IR: 78.4 ; 95\% CI 72.4 to 84.8 ) of match injuries occurred in contact situations. More than half of all injuries (54\%) occurred during a tackle (27\% to the tackler, $23 \%$ to the ball carrier, $4 \%$ unspecified), and $13 \%$ occurred while running (table 8 ).

\section{Discussion}

The main findings of this study in South African professional men's rugby union teams over five seasons of the Super Rugby tournament (2012-2016) were as follows ${ }^{1}$ : the overall incidence of time loss injuries was 10.0 per 1000 player-hours ${ }^{2}$; match injury incidence (99.9 per 1000 hours) was 62 times higher than training injury incidence (1.6 per 1000 hours) 3 ; about $50 \%$ of all players can be expected to suffer a time loss injury each season, with almost a third expected to incur an injury resulting in 8 or more days of time loss and one in eight players sustaining a severe injury ( $>28$ days of time loss) 4 ; the match IB over the 5 -year period was 1796 with a tendency to increase over the 5 -year study period5; the overall incidence of injury between forwards and backs was not significantly different ${ }^{6}$; moderate and severe injuries accounted for $38 \%$ of match injuries and $42 \%$ of training injuries 7 ; the severity profile was similar between match and training injuries $8 ; 50 \%$ of match injuries affected the lower limb 9 ; incidence was highest for the thigh (13.1 per 1000 player-hours), knee (13.1), shoulder/clavicle (12.9) and head/ face $(11.7)^{10}$; the majority of injuries involved muscles/tendons (47\%) and joints/ligaments $(32 \%)^{11}$; there has been an increase in head/face and brain/CNS injury incidence since 2014 ${ }^{12}$; most injuries occur in contact situations (79\%) and more than half occur during a tackle(54\%). 


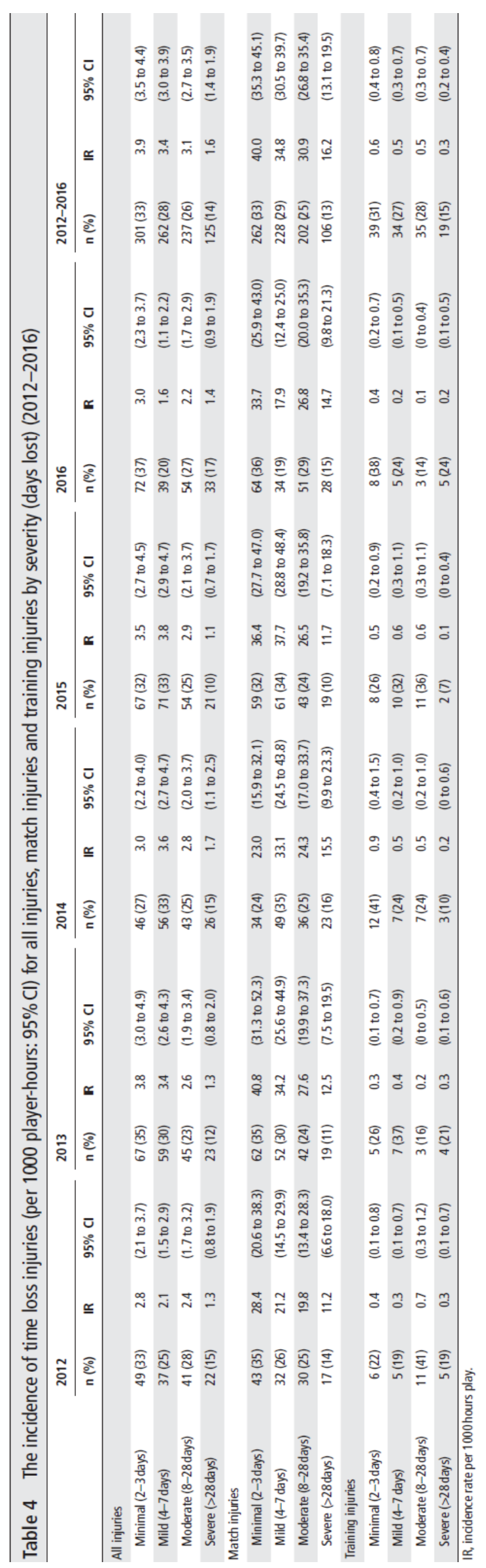



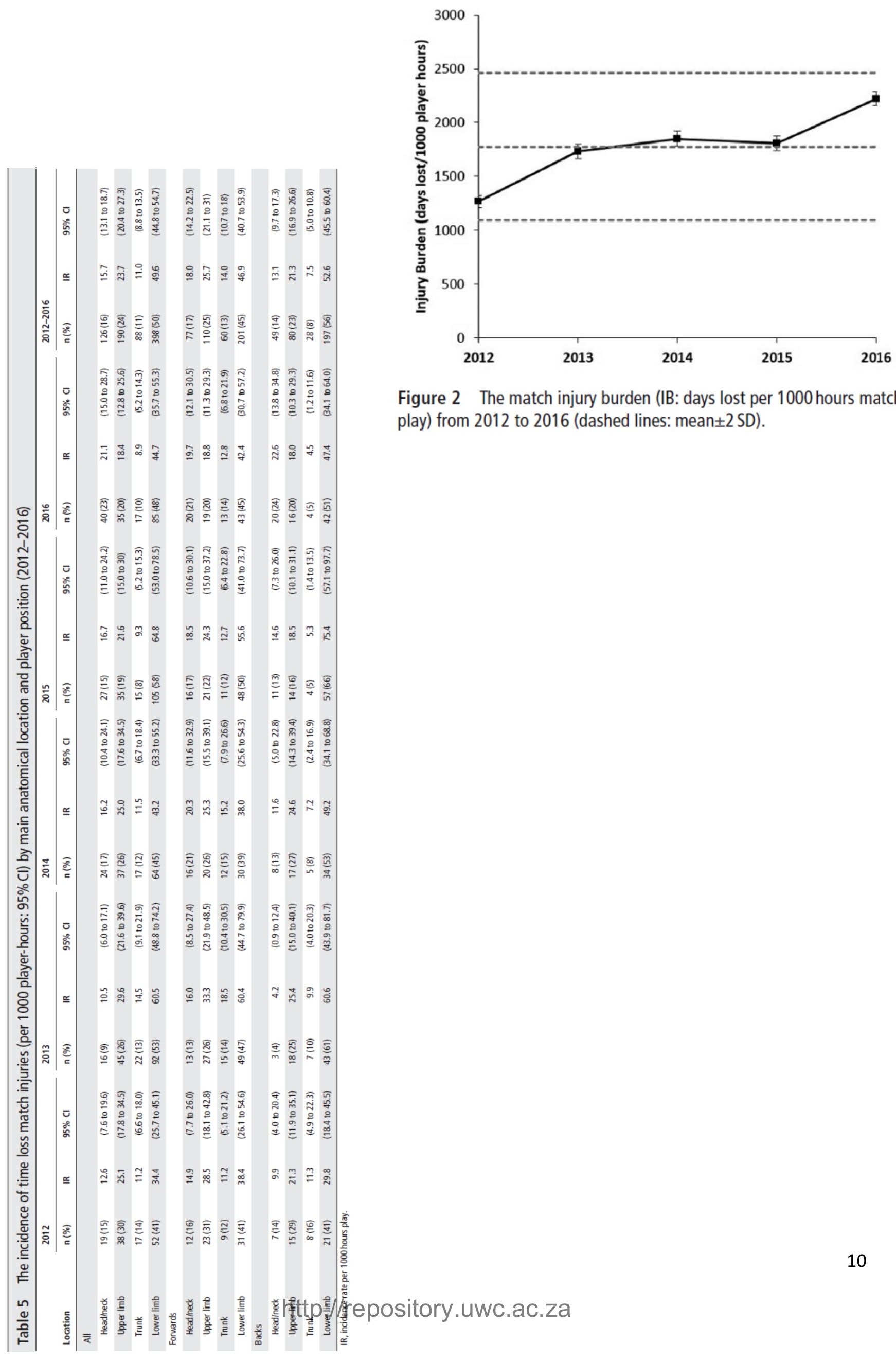

Figure 2 The match injury burden (IB: days lost per 1000 hours match play) from 2012 to 2016 (dashed lines: mean \pm 2 SD). 
This study is the largest prospective study in the Super Rugby tournament to date. In comparison to two previous studies that were conducted in single seasons during the Super Rugby tournament, 56 the match injury incidence among South African teams in the Super Rugby tournament over the past 5 years is considerably higher, although relatively stable at a mean of 99.9 (SD 13.4) per 1000 player-hours, with a minimum of 83 (2012) and a maximum of 115 (2013). Furthermore, over a similar time period, match injury incidence during the England Rugby Premiership competition (from the 2011-2012 to 2015-2016 season) has ranged from 62 (2015-2016) to 91 (2013-2014) per 1000 player-hours, with a mean of 77.4 (SD 10.8). ${ }^{8}$ A metaanalysis of data published before 2011, which was heavily weighted towards figures from the England Rugby Premiership, reported an overall match injury incidence of 81 per 1000 playerhours. ${ }^{1}$ Therefore, our study shows that there is a substantially higher match injury incidence in South African Super Rugby, where each team can expect to incur two injuries per match on average, compared with professional rugby union teams in England, at 1.5 injuries per match. These differences in injury incidence could be related to a complexity of many factors including differences in conditioning levels, injury prevention and management procedures, or travel demands during the tournament. 


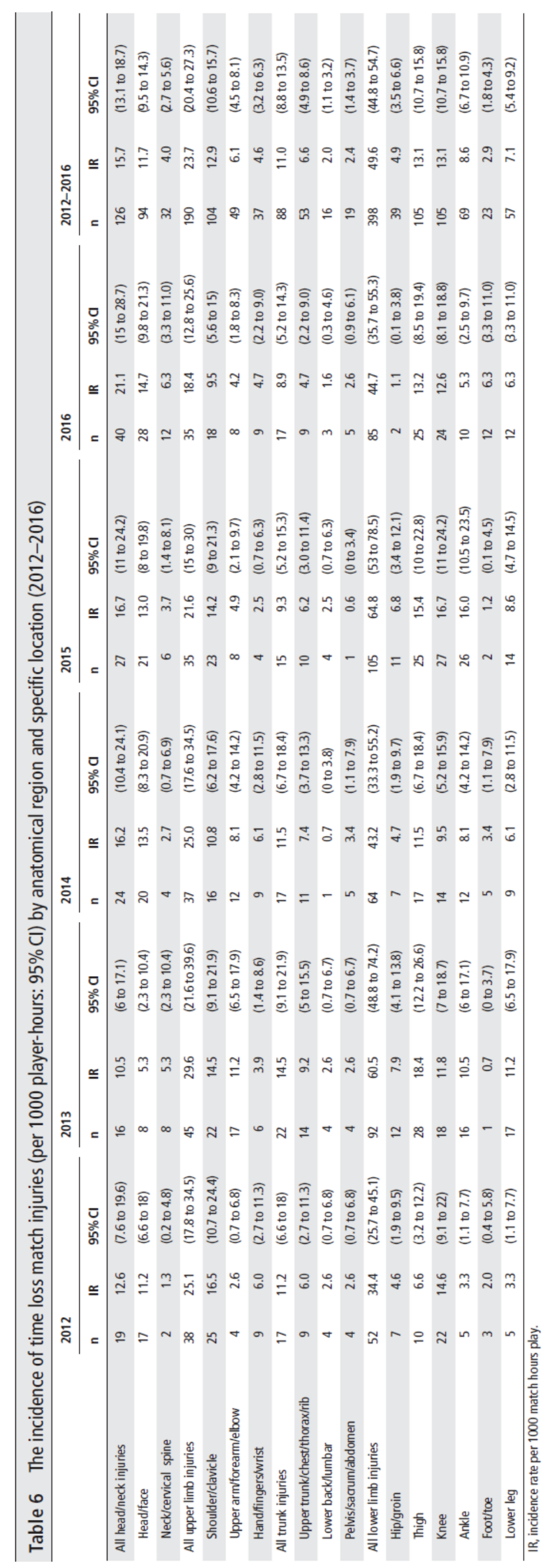




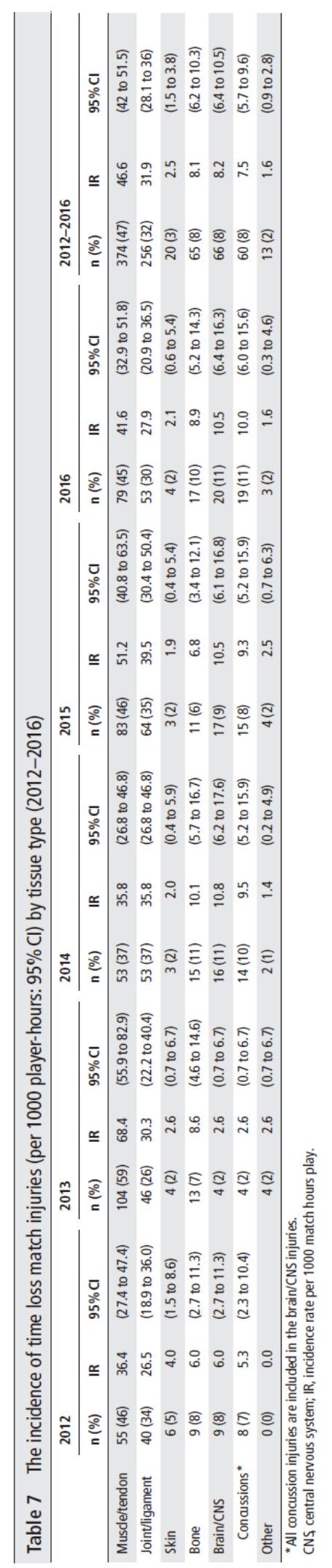




\begin{tabular}{|c|c|c|c|c|c|c|}
\hline & 2012 & 2013 & 2014 & 2015 & 2016 & 2012-2016 \\
\hline Mechanism & $\mathrm{n}(\%)$ & $n(\%)$ & $n(\%)$ & $n(\%)$ & $n(\%)$ & $n(\%)$ \\
\hline Kicking & $3(2)$ & $0(0)$ & $0(0)$ & $2(1)$ & $1(1)$ & $6(1)$ \\
\hline Collision & $6(5)$ & $6(3)$ & $11(8)$ & $23(13)$ & $11(6)$ & $57(7)$ \\
\hline Ruck & $17(13)$ & $18(10)$ & $13(9)$ & $11(6)$ & $18(10)$ & $77(10)$ \\
\hline Running & $9(7)$ & $21(12)$ & $16(11)$ & $28(15)$ & 30 (17) & $104(13)$ \\
\hline Scrum & $7(6)$ & $12(7)$ & $6(4)$ & $11(6)$ & $12(7)$ & $48(6)$ \\
\hline Tackle-unspecified & $4(3)$ & $6(3)$ & $6(4)$ & $16(9)$ & $4(2)$ & $36(4)$ \\
\hline Tackled & $32(25)$ & $56(32)$ & $29(20)$ & $29(16)$ & $42(24)$ & $188(23)$ \\
\hline Tackling & $36(29)$ & $46(26)$ & $41(29)$ & $48(26)$ & $43(24)$ & $214(27)$ \\
\hline Other & $12(10)$ & $10(6)$ & $20(14)$ & $14(8)$ & $16(9)$ & $72(9)$ \\
\hline
\end{tabular}

Compared with our study, a lower incidence (per 1000 match player-hours) was also reported among five South African teams in the 2012 Super Rugby tournament (83.3) and five Australian teams in the 2014 Super Rugby tournament (66.1), where the same overall methodology, including injury and exposure definitions as the present study, was used.5 6 However, we acknowledge that season-on-season variation is not accounted for in the single-season studies, and factors such as team physicians becoming familiar with the data collection procedure in the first year of a study could influence the data collection. Longitudinal data would be required for a valid comparison between our results and other tournaments.

In our study, we show that injuries that resulted in more than 7 days of time loss (moderate and severe injuries) comprised $38 \%$ of all match injuries.

The incidence of moderate (30.9 per 1000 player-hours) and severe (16.1 per 1000 playerhours) match injuries was similar to that reported in a meta-analysis of five published studies (28 and 15 per 1000 player-hours, respectively), ${ }^{1}$ indicating that the higher overall incidence than earlier studies was due to a greater incidence of less severe (mild and minimal) injuries in South African Super Rugby teams. Further- more, although the mean match IB in our study is similar to that reported in the England Professional Rugby Injury Surveillance Project, 8 our data do show a trend towards an increasing match IB over the 5-year period.

This supports more recent data from English and Australian teams where a trend towards increasing injury severity is suggested. 68 However, it is not possible to conduct a direct comparison with these studies due to differences in the reporting of severity, with the English study introducing an additional category for injuries resulting in greater than 84 days of time loss, the Australian study reporting mean time loss in days for various injury descriptors rather than the incidence within each severity category, and in our study a limitation is that the total days lost were reported as estimated days lost at the time of reporting the injury, rather than actual days lost.

In accordance with previous studies, ${ }^{1} 6$ we show that the lower limb was the most commonly injured region, with the thigh (13.1) and knee (13.1) being the two specific locations with the highest match injury incidence per 1000 player-hours. 
The shoulder/clavicle has also previously been shown to be one of the most commonly injured locations and our results support this, with a match incidence of 12.9 per 1000 player-hours. Thigh and shoulder/clavicle injury incidence, in particular, was higher than that reported in Australian Super Rugby teams (8.3 and 8.9 per 1000 player-hours, respectively). Further investigation into the reasons for differences in the incidence of these common injuries may provide guidance for injury risk reduction interventions. We observed an increase in head/face injuries and concussion after 2013. In the England Rugby Premiership, an increase in the incidence of concussion has also been observed over this period. ${ }^{8}$ It is likely that this can be attributed to improved awareness of concussion as a result of educational initiatives and rule changes. The Pitch Side Concussion Assessment protocol, which was trialled since 2013 and became a permanent World Ruby Law in the form of the Head Injury Assessment Tool in 2015, ${ }^{10}$ appears to have increased recognition and reporting of injuries affecting the brain/CNS (mostly concussion) by team medical staff.

The majority (79\%) of match injuries occurred in contact situations, at a high incidence of 78.4 per 1000 player-hours. Given that the tackle is the most frequently occurring event in a match and, by its nature, involves a collision between players, it is perhaps not surprising that more than half (54\%) of all match injuries were reported to occur during the tackle. This figure is consistent with previous studies. ${ }^{1}$ Rule changes and modification of players' technique are two mechanisms that may have an effect on tackle-related injuries. World Rugby has implemented recent law changes, with a specific focus on reducing the risk of head injury during a tackle. Tackle technique features associated with tackle-related injury have been identified in adult professional ${ }^{11}$ and competitive under-18 players. ${ }^{12}$ However, to our knowledge, no targeted interventions and reassessment of injury incidence have been carried out based on these technical recommendations. These data may serve as a baseline for assessing the efficacy of such interventions.

The strengths of this study are that it is the largest prospective study conducted to date during the Super Rugby tournament, compliance of the data collection by the team physicians over the duration of the study has been very high, and consistent methodology, based on the international guidelines, 9 was used. A limitation of the data collection is that we did not record final return to play days and we could therefore only estimate the match IB. In addition, our injury severity data could only be compared with studies that used the same categories of injury severity that were suggestedin theinternational consensus paper on reporting rugby injuries. 9

\section{Conclusion}

In summary, this study adds to the epidemiological under- standing of injury in professional men's rugby union, as such a longitudinal study of this size has not been completed outside of the English Rugby Premiership. The main finding is that there is a higher overall incidence of match injury in South African Super Rugby teams, compared with the English Rugby Premiership and compared with two previous studies during single seasons in the Super Rugby tournament. 


\section{What are the findings?}

- Match injury incidence is higher in South African teams participating in Super Rugby than in previously reported teams and tournaments.

- About $50 \%$ of all players can be expected to suffer a time loss injury each season, with almost a third expected to incur an injury resulting in 8 or more days of time loss and one in eight players sustaining a severe injury ( $>28$ days of time loss).

- Over the 5 years, the mean injury burden (IB) is similar to that reported in other studies, but the tendency over the study period is that the IB is increasing.

- There has been an increase in the incidence of head/face injuries and concussion reported since 2014.

\section{How might it impact on clinical practice in the future?}

- The Super Rugby tournament is associated with a significant risk of injury (50\% players injured during the tournament).

- Medical staff and team management can plan appropriate player management such as increasing the size of squad prior to the tournament.

- Risk factors for injury should be identified, risk management strategies developed and these strategies should be tested in order to reduce the risk of injury, particularly severe injuries, in the Super Rugby tournament.

However, injury patterns are similar to those reported in other studies but injury severity appears to increase. Further analysis of risk factors and mechanisms of the more common and severe injuries is warranted. There is a need to develop evidence-based guidance for injury risk reduction programmes during the Super Rugby tournament and we have taken steps to design and implement such injury prevention programmes. 


\section{Acknowledgements}

The authors acknowledge all team physicians who contributed to data collection $(<2$ years), Marisce Blackaller-Smith for administrative assistance in 2012-2014 and Audrey Janse van Rensburg for administrative assistance in 2015-2016. MPS was employed at the University of Cape Town until end of March 2015 and acknowledges the University of Cape Town in facilitating the data collection in the period 2012-2014. MPS still holds the position of Emeritus Professor at the Faculty of Health Sciences (University of Cape Town, South Africa).

Contributors MPS: responsible for the overall content as guarantor, study concept, study planning, data collection, data interpretation, manuscript (first draft), manuscript editing, facilitating funding. EJ: study planning, data analysis including statistical analysis, data interpretation, manuscript editing. CJR: data interpretation, manuscript drafting and editing. WD: study planning, data collection, data interpretation, manuscript editing. CR: data interpretation, manuscript editing, facilitating funding. RC, AK, JS, OS: data collection, data interpretation, manuscript editing.

Funding This study was funded by the IOC Research Center, South Africa (partial funding), and the South African Rugby Football Union Medical Committee (partial funding).

Competing interests CR is the chairman of the South African Rugby Football Union Medical Committee. The other authors declare no conflicts of interest.

Patient consent Not required.

Ethics approval Ethical approval for the study was obtained from both the research ethics committees of the University of Cape Town (REC numbers 008/2011 and 736/2013) and the University of Pretoria (432/2015).

Provenance and peer review Not commissioned; externally peer reviewed.

Data sharing statement The principal investigator and some members of the research team will explore further detailed injuries and risk factors using the existing data set. 


\section{References}

1. Williams S, Trewartha G, Kemp S, et al. A meta-analysis of injuries in senior men's professional Rugby Union. Sports Med 2013;43:1043-55.

2. Williams S, Trewartha G, Kemp SP, et al. Time loss injuries compromise team success in Elite Rugby Union: a 7-year prospective study. Br J Sports Med 2016;50:651-6.

3. Finch C. A new framework for research leading to sports injury prevention. $J$ Sci Med Sport 2006;9:3-9. Discussion 10.

4. Kemp S, Brooks J, Fuller C. England Rugby premiership injury and training audit. England, 2011.

5. Schwellnus MP, Thomson A, Derman W, et al. More than 50\% of players sustained a time-loss injury ( $>1$ day of lost training or playing time) during the 2012 super rugby union tournament: a prospective cohort study of 17,340 player-hours. Br J Sports Med 2014;48:1306-15.

6. Whitehouse T, Orr R, Fitzgerald E, et al. The Epidemiology of injuries in Australian professional rugby union 2014 super rugby competition. Orthop JSports Med 2016;4:23.

7. Fuller CW, Raftery M, Readhead C, et al. Impact of the International Rugby Board's experimental law variations on the incidence and nature of match injuries in southern hemisphere professional rugby union. SAfr MedJ 2009;99:232-7.

8. Kemp S, Brooks J, West S, et al. England Professional Rugby Injury Surveillance Project: 2015-16 Season Report. $\quad$ England. $2017 . \quad$ (http://www.englandrugby. com/mm/Document/General/General/01/32/25/17/1516_PRISP_Annual_Report_

FINAL(withcontentspage)_English.pdf)

9. Fuller CW, Ekstrand J, Junge A, et al. Consensus statement on injury definitions and data collection procedures in studies of football (soccer) injuries. Br JSports Med 2006;40:193-201.

10. Raftery M, Tucker R. Implementing a worldwide concussion programme: the world rugby strategy to secure player welfare: Aspetar Sports Medicine Journal: Aspetar - Orthopaedic and Sports Medicine Hospital, 2016.

11. Quarrie KL, Hopkins WG. Tackle injuries in professional Rugby Union. AmJ Sports Med 2008;36:1705-16.

12. Burger N, Lambert MI, Viljoen W, et al. Mechanisms and factors associated with tackle-related injuries in South African Youth Rugby Union Players. AmJ Sports Med 2017;45:278-85. 